\title{
Colpocleisis: Do We Need to Consider it More?
}

\author{
Dua A, Elnaggar A*, Bombieri L and Freeman $\mathbf{R}$ \\ University Hospitals Plymouth NHS Trust, UK \\ *Corresponding author: Elnaggar A, University Hospitals Plymouth NHS Trust, UK
}

\begin{tabular}{|c|c|}
\hline ARTICLE INFO & ABSTRACT \\
\hline Received: 幽 September 28, 2019 & Citation: Dua A, Elnaggar A, Bombieri L, Freeman R. Colpocleisis: Do We Need to \\
\hline Published: 幽 October 04, 2019 & Consider it More?. Biomed J Sci \& Tech Res 21(5)-2019. BJSTR. MS.ID.003653. \\
\hline
\end{tabular}

\section{Introduction}

With on-going advances in technology, healthcare and lifestyles, people around the world are living longer on average than they might have in years gone by. In the UK one in every five people (18.2\%) were 65 years or more in 2017 and this is projected to reach around one in every four people (24\%) by 2037 [1]. In the USA, people over 65 years will represent $19 \%$ of population by 2030, whereas in Japan they comprise $40.1 \%$ of the whole population currently [2]. Women more than 80 years of age are the fastest growing segment of society with advanced age, the incidence and prevalence of Pelvic Organ Prolapse (POP) increases and it becomes an increasingly bothersome disorder. In the subgroup of patients 80 years of age or older, the prevalence of POP is reported to be $37 \%$ [3]. However, given the ageing of the "baby boomer" population, a substantial increase is predicted in those seeking treatment for prolapse in the future [4].

Substantial numbers of women in the older age group suffer from complex physical or cognitive disorders, which make complex major surgeries a highly challenging task for both doctors and patients. Options for treatment of POP include non-surgical conservative management, surgical reconstructive surgery and obliterative surgery. In cases of POP, the majority of urogynaecologists consider a vaginal pessary as the first line of treatment for women who are either unable or unwilling to undergo surgical repair. However, it sometimes becomes more difficult to maintain a vaginal pessary either because it won't stay in; causes bleeding or incontinence and some patients are unwilling to tolerate it indefinitely [5]. When a woman cannot remove and reinsert the device herself, the pessary requires a lifelong commitment to visits to a doctor or a nurse every 3-6 months for a pessary change which can sometimes be uncomfortable. This commitment is especially problematic for patients who become unable to drive or who lack social or community nursing support. Maintenance of the pessary becomes more frustrating as the patients becomes more dependent. It is not uncommon for gynaecologists to come across patients with serious complication such as vesico-vaginal or recto-vaginal fistula from a 'neglected' pessary [6]. The fundamental reason for choosing an obliterative procedure such as colpocleisis over vaginal, abdominal, or laparoscopic reconstructive surgery is to treat the prolapse with the least invasive technique in the shortest time. This makes it an ideal procedure for women who do not wish to retain penetrative vaginal sexual function and/or have co-morbidities which are common in this age group. Hysterectomy, which is the commonest surgical procedure for POP, often adds 30 to 80 minutes to the procedure with increased morbidity and should therefore only be performed in older patients if there is a suspected uterine pathology upon initial evaluation or if there is a desire to retain vaginal sexual function [7].

Different techniques of obliterative surgeries have been described including vaginectomy [8] purse-string closure [9] colpocleisis after performing standard anterior and posterior vaginal wall repair [10] purse-string closure of enterocele followed by approximation of perivesical and rectovaginal fascia, high levator plication [11] and LeFort colpocleisis [12] where a bridge of tissue is created between the anterior and posterior vaginal wall to stop the vault prolapse from protruding. A partial colpocleisis, performed using the LeFort technique with limited dissection, has become the most popular obliterative approach [7]. First described in 1877, this procedure still remains underused and unpopular amongst surgeons, despite excellent success rates and patient satisfaction. This is supported by the findings from a national surgical database of the British Society of Urogynaecology which shows that Colpocleisis comprised less than $5 \%$ of all procedures performed for POP in the UK in the last few years. 
It is believed that Colpocleisis may adversely affect body image, cause regret for loss of coital function, and, ultimately, could result in high percentage of patients' non-satisfaction and regret [13]. However, numerous studies have refuted this claim. One of the largest studies including 278 women post-colpocleisis reported that approximately $97 \%$ of patients were satisfied with their decision to have vaginal closure for the treatment of prolapse and none of them regretted their decision. In addition, total body image scores improved significantly following the surgery [14]. Furthermore, there are no adverse effects reported on bladder and bowel function following a colpocliesis [15]. Another factor that may contribute to the underutilisation of colpocleisis is the paucity of long-term information and outcomes reported on the obliterative LeFort procedure. Most of the information on colpocleisis has come from case series with poorly defined postoperative outcome measures and follow-ups [16]. Although the definition of success is not clear in some series, the reported success rate has always exceeded $90 \%$ over the past three decades with low recurrence rates $[17,18]$.

Longer term follow-up of up to 6 years also shows a low recurrence risk of further POP and no regret due to loss of sexual function [19]. Colpocleisis has the advantages of a shorter operating time, fewer complications, and the possibility of performing it under local anaesthesia. In addition, it is characterised by shorter hospitalization, quick recovery, higher success rate, and low rate of regret [7]. Sung et al. reported that obliterative procedures have a lower risk of complications than reconstructive procedures for treating prolapse for patients with extreme old age $(17.0 \%$ vs. $24.7 \%$ ) [16]. Because of these advantages, an increasing number of older but otherwise healthy women are choosing obliterative vaginal surgery. Colpocleisis is a viable choice for frail, elderly women who have stage III or IV POP who do not desire or foresee the possibility of future vaginal intercourse, and if they have medical co- morbidities or previous history of multiple surgical failures. Such women represent a surprisingly high percentage of the elderly population. A community-based survey found that $78 \%$ of married women 70 to 79 years old are not sexually active [20].

A recent meta- analysis of vaginal obliterative procedures for pelvic organ prolapse showed that both Le Fort and total colpocleisis are effective procedures for surgical treatment of advanced pelvic organ prolapse with relatively low complication rates and high patient satisfaction. Loss of coital function is rarely a reason for regret. In addition, Hysterectomy at the time of colpocleisis is rarely required in most patients with procidentia [21]. UK professional bodies including the Royal college of Obstetrician Gynaecologists (RCOG) and National Institute for Clinical Excellence (NICE) support the use of colpocleisis for women who have vault or uterine prolapse and who do not intend to have intercourse and who have physical conditions/co-morbidities that may put them at increased risk of intra-operative and postoperative complications. (RCOG green top guidelines for management of post-hysterectomy vault prolapse 2015; NICE guidelines for Urinary Incontinence and Pelvic Organ Prolapse: Management 2019).

The British Society ofUrogynaecology (BSUG) data base (January 2013 to May 2019) showed that 1212 colpocleisis operations have been performed nationally in the UK. Of those 1212 operations, 129 were performed in our unit at University Hospitals Plymouth (10.6\%). The mean age for the group of those 129 patients, was 77 years (Range 67-89). No major intraoperative complications were seen for any of our patients. 2 cases had postoperative bleeding that needed further suturing without the need for blood transfusion, and one case had short-term voiding dysfunction with the need for catheterisation for ten days postoperatively). Only 3 cases $(2.3 \%)$ had recurrence after the surgery which has been treated successfully by a 're-do' colpocleisis. Despite the current evidence and obvious benefits, a recent survey of a urogynaecologist-predominant group in the USA showed a wide variation in the current practice of colpocleisis with lack of standard treatment guidelines for this safe and effective procedure [22].

In conclusion, Colpocleisis is a simple and effective procedure for the surgical management of POP in older age group of women who are not sexually active. It is well accepted by patients with high satisfaction rates and low levels of complications. It is underutilised by many units across the UK and more awareness about the procedure is in our patients' best interest.

\section{References}

1. (2014) Administration for Community Living, Administration on Aging. Aging statistics.

2. W He, D Goodkind, P Kowal (2016) An aging world: 2015 US Census Bureau pp. 1-165.

3. Morley GW (1996) Treatment of uterine and vaginal prolapse. Clin Obstet Gynecol 39(4): 959-969.

4. Wu JM, Kawasaki A, Hundley AF, Dieter AA, Myers ER, Sung VW (2011) Predicting the number of women who will undergo incontinence and prolapse surgery, 2010 to 2050. Am J Obstet Gynecol 205(3): 230. e231-e235.

5. Cundiff GW, Weidner AC, Visco AG, Bump RC, Addison WA (2000) A survey of pessary use by members of the American Urogynecologic Society. Obstet Gynecol 95(6 Pt 1):931-935.

6. Esin S, Harmanli OH (2008) Large vesicovaginal fistula in women with pelvic organ prolapse: the role of colpocleisis revisited. Int Urogynecol J Pelvic Floor Dysfunct 19: 1711-1713.

7. Harmanli MD (2009) Colpocleisis: a simple, effective and underutilized procedure for pelvic organ prolapse. OBG Manag 21(6): 19-28.

8. Hoffman MS, Cardosi RJ, Lockhart J, Hall DC, Murphy SJ (2003) Vaginectomy with pelvic herniorrhaphy for prolapse. Am J Obstet Gynecol 189: 364-370.

9. DeLancey J0, Morley GW (1997) Total colpocleisis for vaginal eversion. Am J Obstet Gynecol 176:1228-32.

10. Harmanli OH, Dandolu V, Chatwani AJ, Grody MT (2003) Total colpocleisis for severe pelvic organ prolapse. J Reprod Med 48(9): 703-706.

11. Von Pechmann WS, Mutone M, Fyffe J, Hale DS (2003) Total colpocleisis with high levator plication for the treatment of advanced pelvic organ prolapse. Am J Obstet Gynecol 189(1): 121-126. 
12. Pelosi MA, Pelosi MA (1998) Transvaginal needle suspension with LeFort colpocleisis for stress incontinence and advanced uterovaginal prolapse in a high-risk patient. J Am Assoc Gynecol Laparosc 5(2): 207 11.

13. 13.Park JY, Han SJ, Kim JH, Chun KC, Lee TS (2019) Le Fort partia colpocleisis as an effective treatment option for advanced apical prolapse in elderly women. Taiwanese Journal of Obstetrics and Gynecology 58(2): 206-211.

14. Wang X, Chen Y, Hua K (2017) Pelvic symptoms, body Image, and regret after LeFort Colpocleisis: a long-term follow-up. J Minim Invasive Gynecol 24(3): 415-419.

15. Vij M, Bombieri L, Dua A, Freeman R (2014) Long-term follow-up after colpocleisis: regret, bowel, and bladder function. International urogynecology journal 25(6): 811-815.

16. Abbasy S, Kenton K (2010) Obliterative procedures for pelvic organ prolapse. Clinical obstetrics and gynecology 53(1): 86-98.

\section{ISSN: 2574-1241}

DOI: 10.26717/BJSTR.2019.21.003653

Elnaggar A. Biomed J Sci \& Tech Res

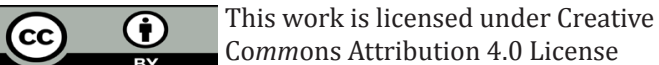

Submission Link: https://biomedres.us/submit-manuscript.php
17. Sung VW, Weitzen S, Sokol ER, Rardin CR, Myers DL (2006) Effect of patient age on increasing morbidity and mortality following urogynecologic surgery. American journal of obstetrics and gynecology. 2006 May 1;194(5): 1411-1417.

18. Winkelman WD, Haviland MJ, Elkadry EA (2018) Long-term Pelvic Floor Symptoms, Recurrence, Satisfaction, and Regret Following Colpocleisis. Female Pelvic Med Reconstr Surg

19. Patel D, Gillespie B, Foxman B (2003) Sexual behavior of older women: results of a random-digit-dialing survey of 2,000 women in the United States. Sex Transm Dis 30(3): 216-220.

20. Buchsbaum GM, Lee TG (2017) Vaginal obliterative procedures for pelvic organ prolapse: a systematic review. Obstetrical \& gynecological survey $72(3): 175-183$

21. Jones K, Wang G, Romano R, St Marie P, Harmanli O (2017) Colpocleisis: a survey of current practice patterns. Female pelvic medicine \& reconstructive surgery 23(4): 276-280.

$\begin{array}{ll}\text { BIOMEDICAL } & \text { Assets of Publishing with us } \\ \text { RESEARCHES } & \text { - Global archiving of articles } \\ & \text { - Immediate, unrestricted online access } \\ & \text { - Rigorous Peer Review Process } \\ \end{array}$

